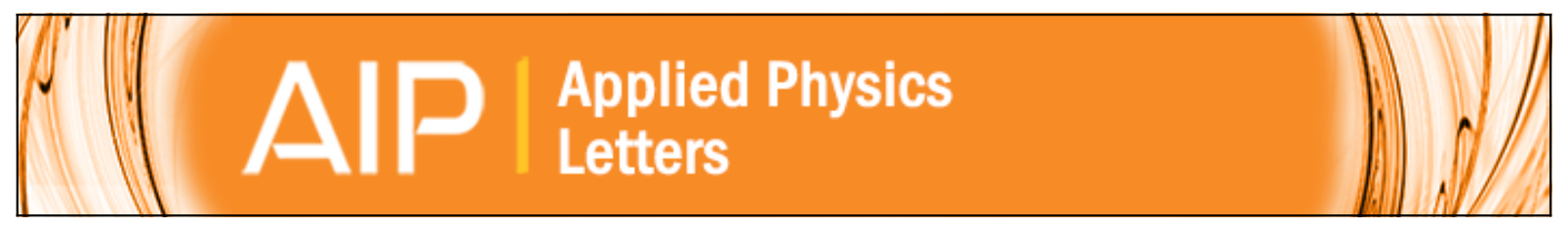

Comparison of experimental and theoretical gain-current relations in GalnP quantum well lasers

P. M. Smowton, P. Blood, and W. W. Chow

Citation: Applied Physics Letters 76, 1522 (2000); doi: 10.1063/1.126083

View online: http://dx.doi.org/10.1063/1.126083

View Table of Contents: http://scitation.aip.org/content/aip/journal/apl/76/12?ver=pdfcov

Published by the AIP Publishing 


\title{
Comparison of experimental and theoretical gain-current relations in GalnP quantum well lasers
}

\author{
P. M. Smowton ${ }^{\mathrm{a})}$ and P. Blood \\ Department of Physics and Astronomy, Cardiff University, P.O. Box 913, Cardiff, Wales, \\ CF2 3YB, United Kingdom
}

W. W. Chow

Sandia National Laboratories, Albuquerque, New Mexico 87185-0601

(Received 22 November 1999; accepted for publication 28 January 2000)

\begin{abstract}
We compare the results of a microscopic laser theory with gain and recombination currents obtained from experimental spontaneous emission spectra. The calculated absorption spectrum is first matched to that measured on a laser, ensuring that the quasi-Fermi levels for the calculation and the experiment (spontaneous emission and gain) are directly related. This allows us to determine the inhomogeneous broadening in our experimental samples. The only other inputs to the theory are literature values of the bulk material parameters. We then estimate the nonradiative recombination current associated with the well and waveguide core from a comparison of measured and calculated recombination currents. (C) 2000 American Institute of Physics. [S0003-6951(00)04412-0]
\end{abstract}

AlGaInP based quantum well lasers emitting between 620 and $690 \mathrm{~nm}$ are of interest due to an ever increasing range of applications, including digital versatile disk (DVD) and photodynamic therapy. At the short end of the wavelength range it is still difficult to design lasers to meet all the performance requirements, particularly threshold current and efficiency. To aid the design we need to have a good description of the gain and recombination process and, in addition, to understand which of the possible recombination mechanisms are significant in these devices. In this letter we make a detailed comparison of the results of a microscopic laser theory with gain and recombination currents obtained from an analysis of experimental spontaneous emission spectra. To begin the calculated absorption spectrum is matched to that measured on the laser chips, enabling us to make accurate comparisons between theory and experiment in terms of internal quasi-Fermi level separation. Through this analysis we determine the inhomogeneous broadening in the laser samples to be $10 \mathrm{meV}$. The only other input parameters represent the bulk material band structure and these are obtained from the literature. Having fixed all parameters, a comparison between the calculated and measured device current enables us to determine the carrier loss rate due to nonradiative recombination in the well and waveguide core region.

The structure we have chosen to investigate consists of a 6.8-nm-wide, compressively strained, $\mathrm{Ga}_{0.41} \mathrm{In}_{0.59} \mathrm{P}$ quantum well set in an $\left(\mathrm{Al}_{0.5} \mathrm{Ga}_{0.5}\right)_{0.51} \mathrm{In}_{0.49} \mathrm{P}$ waveguide core and clad with $\left(\mathrm{Al}_{0.7} \mathrm{Ga}_{0.3}\right)_{0.51} \mathrm{In}_{0.49} \mathrm{P}$. These layers emit at $670 \mathrm{~nm}$. We observe that devices of length between 250 and $750 \mu \mathrm{m}$ have threshold currents that increase in a linear fashion with temperature up to about $350 \mathrm{~K}$, with an exponential type increase becoming evident above $350 \mathrm{~K}$ for the shorter devices. The measured external differential efficiency is also constant up to approximately $350 \mathrm{~K}$. These observations lead us to believe that the thermally activated loss of carriers to

${ }^{a)}$ Electronic mail:smowtonpm@cf.ac.uk the p-cladding layers is small in these structures below 350 $\mathrm{K}$. We have used devices of length 450 and $750 \mu \mathrm{m}$ and have performed the measurements that follow at room temperature to separate the behavior we wish to examine from effects due to thermally activated leakage, which has already been shown to be important in GaInP/AlGaInP devices. ${ }^{1,2}$

We have measured spontaneous emission spectra through a 4 - $\mu \mathrm{m}$-wide opening in the top contact of the 50$\mu \mathrm{m}$-wide, $450-\mu \mathrm{m}$-long oxide stripe lasers. By measurement of the wavelength of the laser line and the slope of the spontaneous emission spectrum, we were able to determine the quasi-Fermi level separation at threshold. ${ }^{3}$ This information is used in the relationship between gain and spontaneous emission ${ }^{4}$ to determine the gain spectrum. The measurements were corrected for the system spectral response but since an unknown fraction of the total spontaneous emission is collected, the spontaneous emission spectra and the derived gain spectra are not given in absolute units. We converted them into real units by determining the threshold loss (or gain $=1917 \mathrm{~cm}^{-1}$ for the $450-\mu \mathrm{m}$-long devices). The internal optical mode loss $\left(\alpha_{i}\right)$ was determined from measurements of the external differential efficiency as a function of device length $\left(\alpha_{i}=7.0 \pm 1 \mathrm{~cm}^{-1}\right)$. The mirror loss $\left(\alpha_{m}=27.5 \mathrm{~cm}^{-1}\right)$ and optical confinement factor $(\Gamma=1.8 \%)$ were determined from the refractive index values for the $\left(\mathrm{Al}_{0.7} \mathrm{Ga}_{03}\right) \mathrm{InP}$ and $\left(\mathrm{Al}_{0.5} \mathrm{Ga}_{05}\right) \mathrm{InP},{ }^{5}$ the aluminum contents being confirmed by photovoltage absorption spectroscopy. ${ }^{6}$ The points in Fig. 1 show the gain spectra measured from a sample at different excitation levels.

The gain calculation was performed by solving the semiconductor Bloch equations, with collision effects treated at the level of quantum kinetic equations. The details of the calculations are given in several letters. ${ }^{7,8}$ This approach has several advantages over the more familiar gain calculations based on the relaxation rate approximation. It eliminates the dephasing rate as a free parameter. It also includes contributions from nondiagonal Coulomb correlations, which are found to be important in describing the experimental shape 


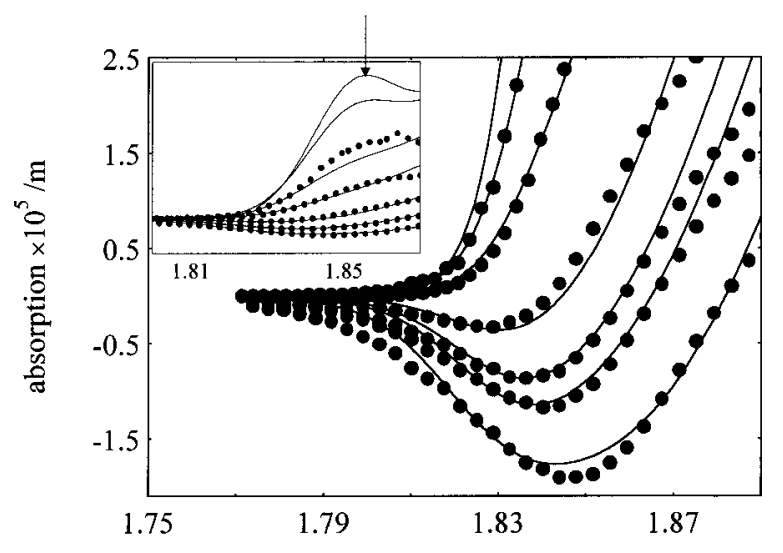

energy /eV

FIG. 1. Room temperature gain/absorption spectra (dots) measured at current densities between 40 and $960 \mathrm{~A} / \mathrm{cm}^{2}$. The calculated spectra (lines) are for carrier densities between $6 \times 10^{15}$ and $3.6 \times 10^{16} \mathrm{~m}^{-2}$. The inset shows the absorption portion of the spectra-the arrow indicating the energy of the 1st electron to heavy-hole transition as measured by photovoltage absorption spectroscopy.

and carrier density dependence of gain spectra. ${ }^{9}$

The inputs to the gain calculations are the band-structure properties, specifically the electron and the hole energy dispersions, as well as the optical dipole matrix elements. These quantities for a $\mathrm{GaInP} /(\mathrm{AlGa}) \mathrm{InP}$ strained quantum well were computed using a $6 \times 6$ Luttinger-Kohn Hamiltonian and the envelope approximation. ${ }^{10}$ Input parameters to the band-structure calculations were the bulk material parameters. ${ }^{11,12}$

To ensure that the inputs to the calculation represent the measured devices we matched the results of the calculation for low carrier density $\left(9 \times 10^{22} \mathrm{~m}^{-3}\right)$ with the energy $(1.855$ $\mathrm{eV}$ ) of the excitonic absorption peak measured by photovoltage absorption spectroscopy on the lasers themselves ${ }^{6}$ (arrow in the inset of Fig. 1).

The experimental results are likely to contain the effects of inhomogeneous broadening due to well width or alloy fluctuations. To take account of these effects, we performed a statistical average of the homogeneous gain and absorption spectra, with a weighting described by a normal distribution that is characterized by an inhomogeneous broadening width. ${ }^{13}$ Inhomogeneous broadening produces a reduction and a red shift of the peak gain. Figure 2 shows the peak gain versus chemical potential separation for inhomogeneous broadening of 0,10 , and $20 \mathrm{meV}$. The solid points represent the experimental results corresponding to the data in Fig. 1. An inhomogeneous broadening of $10 \mathrm{meV}$ best reproduces the experimental gain peak values. This value corresponds to (for example) a $\mathrm{Ga}$ variation in the quantum well of about one percentage point $\left(\mathrm{Ga}_{x} \mathrm{In}_{1-x} \mathrm{P}\right.$, where $\left.x=0.41 \pm 0.01\right)$.

The calculated spectra (lines) in Fig. 1 contain an inhomogeneous broadening width of $10 \mathrm{meV}$ and follow the shape and relative magnitudes of the experimental data. They also correctly predict the separation between the peak gain energy and the peak in the absorption spectrum due to the first electron to heavy-hole transition over a large range of injection level. This process is only possible because the

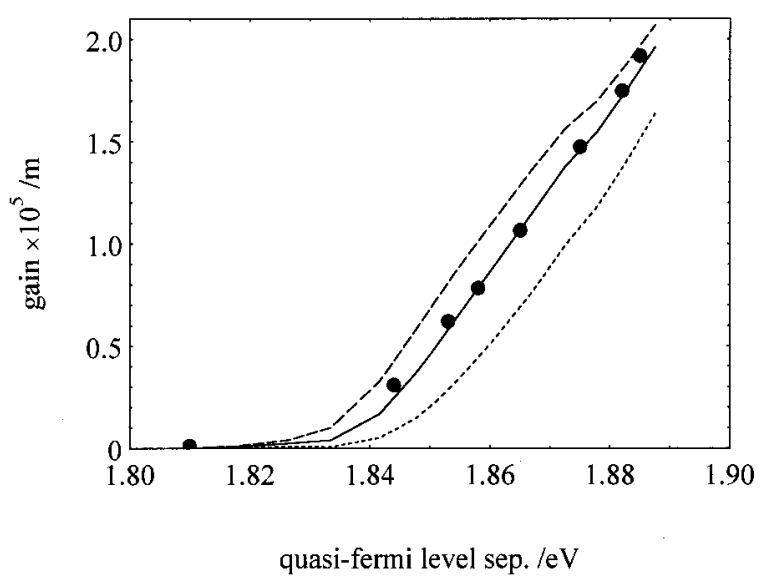

FIG. 2. Peak gain vs quasi-Fermi level separation as measured (solid symbols) and calculated using homogeneous broadening only (dashed line) and for inhomogeneous broadening of 10 (solid line) and $20 \mathrm{meV}$ (dotted line).

quasi-Fermi level separation in theory and experiment are both known for each value of gain.

In addition to determining the transverse electric (TE) peak gain from measurement of the spontaneous emission through the top contact it is also possible to determine the recombination rate associated with the measured TE spontaneous emission by integrating the spontaneous emission spectrum over energy. ${ }^{3}$ The result is given by the dots in Fig. 3 . Theoretically, we obtain this recombination rate by using the calculated gain spectra (Fig. 1) and the same phenomenological relationship between the spontaneous emission spectrum and gain spectrum used in the experiment. ${ }^{4}$ Integrating the spontaneous emission spectrum over energy gives the spontaneous emission rate, $w_{\mathrm{sp}}(N, T)$, in units of $\mathrm{m}^{-3} \mathrm{~s}^{-1}$. Theoretically we may determine either the TE part of the spontaneous recombination rate as shown in Fig. 3 or the total recombination rate by summing the spontaneous emission of both polarizations. This total is the injection current density for a device that has an internal efficiency of $100 \%$. The good agreement between the experimental and theoretical TE spontaneous emission rates as shown in Fig. 3 leads us to believe that the calculated total spontaneous emission rate is also a good representation of that in the real device.

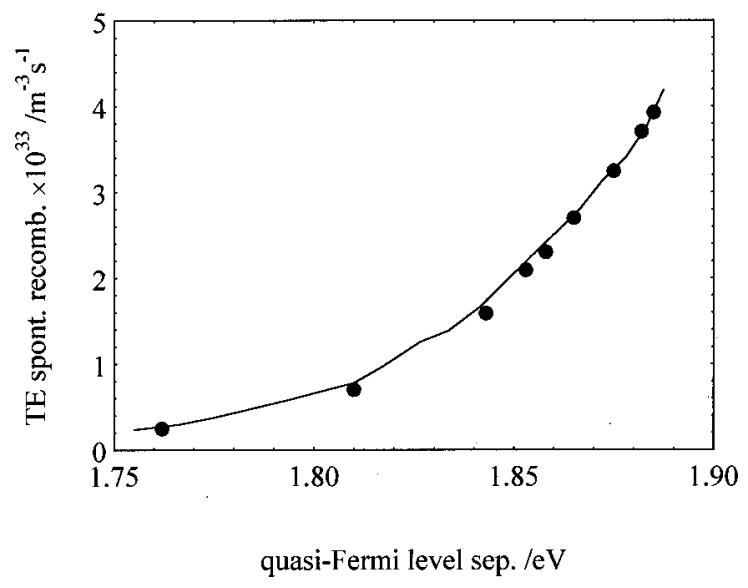

FIG. 3. Theoretical (calculated from the gain spectra, as described in the text) (line) and experimental (solid points) integrated TE polarized spontaneous emission spectra. The experimental data has been scaled by the same factor as the gain spectra. 


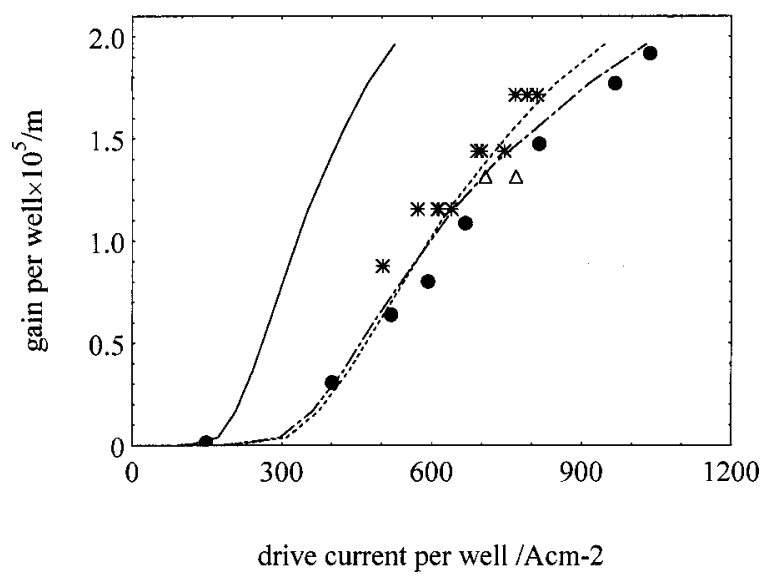

FIG. 4. Peak gain vs calculated total spontaneous current density (line) and experimental drive current for one well, $450-\mu \mathrm{m}$-long device (filled circles), one well, $750-\mu \mathrm{m}$-long device open triangles and 2 well, $450-\mu \mathrm{m}$-long device (stars). The calculated data reflects the fundamental lower limit for a device with an internal efficiency of $100 \%$. The dotted line includes an internal efficiency of 55\%. The dashed line is calculated for a quantum well internal efficiency of $75 \%$ and a nonradiative lifetime in the barrier of $15 \mathrm{ps}$.

We have confirmed that the calculation correctly describes the peak gain (and spectrum) and spontaneous recombination rate in our devices. We now make use of a comparison of the calculated gain-current curve with that derived experimentally to estimate the nonradiative recombination rate within the quantum well and waveguide core of the device.

Figure 4 shows the peak gain versus total (TE and TM) spontaneous emission contribution to the current density (solid line), for the $6.8 \mathrm{~nm} \mathrm{Ga}_{0.41} \mathrm{In}_{0.59} \mathrm{P} /\left(\mathrm{Al}_{0.5} \mathrm{Ga}_{0.5}\right)_{0.51} \mathrm{In}_{0.49} \mathrm{P}$ quantum well. The spontaneous emission current is given by $J_{\mathrm{sp}}=e L_{z} w_{\mathrm{sp}}(N, T)$ per unit area, where $e$ is the electron charge, and $L_{z}$ is the quantum well width. This curve represents the theoretical minimum limit to the threshold current density for a given threshold gain, $G_{\mathrm{th}}=G_{\mathrm{pk}}$. The solid circles are the experimental results obtained from the gain spectra of Fig. 1 and the actual device drive current density (assuming current spreading of 20\%). Further values were obtained from measurements of the threshold loss (gain) versus threshold current for 750- $\mu$ m-long devices (triangles) and on devices which are nominally identical except for having two quantum wells in the active region (stars). In the case of the two well samples the results are plotted in terms of gain and current per well and the threshold loss (gain) was determined in a similar fashion to that described above. The experimental data points for the 450 and $750 \mu \mathrm{m}$ single well devices lie on the same curve and the two well device measurements are similar but both show a large disparity with the calculated results due to additional nonradiative processes. The dotted line of Fig. 4 is the gain plotted as a function of the sum of the calculated radiative recombination current and a nonradiative contribution from the quantum well where we have assumed that this can be represented by an overall internal efficiency (not the differential efficiency) of $55 \%$. The similarity of shape between the experimental data and this theoretical value calculated using a currentindependent efficiency also suggests that any thermally acti- vated loss process does not dominate the recombination in these samples at $300 \mathrm{~K}$. To estimate whether this $55 \%$ efficiency is reasonable we deduce from the calculated total radiative recombination rate at threshold $\left(4.8 \times 10^{33} \mathrm{~m}^{-3} \mathrm{~s}^{-1}\right)$ and the calculated carrier density at threshold $\left(6.8 \times 10^{24}\right.$ $\mathrm{m}^{-3}$ ) that the radiative lifetime at threshold is $1.4 \times 10^{-9} \mathrm{~s}$. A nonradiative lifetime of $1.2 \times 10^{-9} \mathrm{~s}$ is necessary to produce an efficiency of 55\% within the well. A more likely scenario is that part of the nonradiative recombination is due to loss of carriers from the quantum well and part due to nonradiative recombination within the quantum well. For example an internal efficiency in the quantum well of $75 \%$ and a lifetime in the barrier of $15 \mathrm{ps}$ (the carrier density in the barrier is calculated assuming thermal equilibrium between quantum well and barrier) also reproduces the experimental result (dashed line). The exact balance between these two processes is uncertain, however it is necessary to include a quantum well (or current independent) internal efficiency of at most $\approx 75 \%$ in order to match the recombination current at low values of gain. The comparison indicates that there are still significant improvements to be made in these devices with regard to nonradiative recombination within the quantum well and also in the barrier material alongside the quantum well.

In summary we have demonstrated good agreement between experimental gain spectra and TE radiative recombination rates and those produced using a microscopic semiconductor laser theory. By comparing the calculated gain and total radiative recombination current with the measured threshold losses and device drive current we have shown that a significant proportion of the total laser drive current is due to nonradiative recombination within the quantum well and waveguide core.

This work was supported by the Engineering and Physical Sciences Research Council (U.K.) GR/M70698 and the U. S. Department of Energy under Contract No. DE-AC0494AL85000.

${ }^{1}$ D. P. Bour, D. W. Treat, R. L. Thornton, R. S. Geels, and D. F. Welch, IEEE J. Quantum Electron. 29, 1337 (1993).

${ }^{2}$ S. A. Wood, P. M. Smowton, C. H. Molloy P. Blood, D. J. Somerford, and C. C. Button, Appl. Phys. Lett. 74, 2540 (1999).

${ }^{3}$ P. Blood, A. I. Kucharska, J. P. Jacobs, and K. Griffiths, J. Appl. Phys. 70, 1144 (1991).

${ }^{4}$ C. H. Henry, R. A. Logan, and F. R. Merrit, J. Appl. Phys. 51, 3042 (1980).

${ }^{5}$ P. M. Smowton and P. Blood, in Strained Quantum Wells and Their Applications, edited by M. O. Manasreh (Gordon and Breach, Amsterdam, 1997).

${ }^{6}$ P. M. Smowton, P. Blood, P. C. Mogensen, and D. P. Bour, Int. J. Optoelectron. 10, 383 (1996).

${ }^{7}$ W. W. Chow and S. W. Koch, Semiconductor-Laser Fundamentals: Physics of the Gain Materials (Springer, Berlin, 1999).

${ }^{8}$ W. W. Chow, M. Kira, and S. W. Koch, Phys. Rev. B 60, 1947 (1999).

${ }^{9}$ W. W. Chow, P. M. Smowton, P. Blood, A. Girndt, F. Jahnke, and S. W. Koch, Appl. Phys. Lett. 71, 157 (1997).

${ }^{10}$ J. M. Luttinger and W. Kohn, Phys. Rev. 97, 869 (1955).

${ }^{11}$ O. Madelung, Landolt-Bornstein Numerical Data and Functional Relationships in Science and Technology (Springer, Berlin, 1982), Vol. 17a.

${ }^{12} \mathrm{~S}$. Adachi, Physical Properties of III-V Semiconductor Compounds (Wiley, New York, 1992).

${ }^{13}$ W. W. Chow, A. F. Wright, A. Girndt, F. Jahnke, and S. W. Koch, Appl. Phys. Lett. 71, 2608 (1997). 